\title{
The Influence of Initial Carbonate Concentration on the Folin-Ciocalteu Micro-Method for the Determination of Phenolics with Low Concentration in the Presence of Methanol: A Comparative Study of Real-Time Monitored Reactions
}

\author{
Nunzia Cicco ${ }^{1}$, Vincenzo Lattanzio ${ }^{2}$ \\ ${ }^{1}$ Institute of Methodologies for Environmental Analysis, National Research Council, Tito Scalo, Italy \\ ${ }^{2}$ Department of Agro-Environmental Sciences, Chemistry and Crop Protection, University of Foggia, Foggia, Italy \\ E-mail: cicco@imaa.cnr.it \\ Received August 17, 2011; revised September 19, 2011; accepted October 8, 2011
}

\begin{abstract}
During the Folin Ciocalteu (F-C) micro-assay for the determination of phenolics in the presence of methanol, fine solids can form. In a previous paper, we hypothesized that the interference from alcohol on the F-C reaction can be minimized depending on the particular procedure used to reach the alkalinity condition. In order to demonstrate our hypothesis we studied, by spectrophotometrically monitoring, the time-behaviour of the reactions carried out in the presence of different methanol concentrations at the same alkalinity condition from two protocols. The results showed that the interfering effect of methanol on the F-C micro-method can be affect and even prevented depending on working conditions. In particular, the formation of fine solids can be delayed, slowed down and prevented depending on the initial carbonate concentration used. We have explained why the initial carbonate concentration, used to reach the final alkalinity condition, plays an important role in the F-C reaction carried out in the presence of methanol. Moreover, the results from real-time monitoring showed that, differently from traditional F-C procedure, our procedure allows us to carry out the F-C micro method in the presence of $6 \%$ methanol, as an extreme concentration, reading the absorbance at real time $24 \mathrm{~min}$. The real-time monitoring of absorbance can be considered as a useful means to explore the effect of other parameters on precipitate formation caused by the presence of methanol in the F-C reaction.
\end{abstract}

Keywords: F-C Micro-Method, Phenolics, Carbonate Influence, Methanol Effect

\section{Introduction}

Phenolic compounds, widely distributed in plants and food plants, are thoroughly studied by different researchers because of their health beneficial properties $[1,2]$.

Many analytical procedures have been developed to study polyphenolic compounds, in any case, a quantification study is always undertaken as a preliminary step. Although the chromatographic methodology is considered as the most important analytical tool for the quantitative determination of phenolics, the traditional colorimetric methodology is frequently employed for this purpose because of its easier availability in many laboratories.

The colorimetric method generally used is the Folin
Ciocalteu (F-C) one that consists of a rapid oxidation reaction of phenols by using alkali, generally sodium carbonate, which yields an appreciable concentration of the phenolate ions. The phenolates reduce the yellow F-C reactive changing it into a blue pigment, spectrophotometrically measured. Due to the complexity of the competing reactions involved in the $\mathrm{F}-\mathrm{C}$ method, reaction equilibrium is fairly unstable and it is not easy to find the exact conditions for the assay [3]. In order to find the optimal conditions to accomplish the assay, only few authors [3-6] widely studied the influence of parameters such as temperature, reaction time, amount of $\mathrm{F}-\mathrm{C}$ reactive and initial as well as final concentration of alkali. Although the optimized conditions by Singleton are gen- 
erally used [3], different variations in the F-C method are reported in literature [7-13].

However, even though this colorimetric method is often used for the determination of phenolic content in numerous and complex samples, the many insuperable difficulties involved bring its usefulness into question. In fact, due to the lack of selectivity which is peculiar to this method, some substances react with the yellow reactive causing an overestimation of the phenolic content. Moreover, an important F-C method drawback/limitation is that under some conditions precipitates can form. Consequently, it is necessary to resort to additional techniques, such as filtration or centrifugation, which are time-consuming and can sometimes reduce the blue colour yield [3].

In order to prevent the precipitate formation, Singleton and Rossi [3] suggest accomplishing the assay at room temperature and not exceeding the 3\% concentration of the sodium carbonate in the final mixture, although the initial carbonate solution to be used may be relatively concentrated (20\%). Moreover, to obtain reproducible results, Singleton et al. [14] suggest not exceeding the 1\% alcoholic concentration in the final reaction mixture.

In this regard, a sample dilution is carried out to avoid problem of precipitate formation but, sometimes, no dilution of alcoholic extracts can be carried out because of their low phenolic content.

In a recent paper [7], aimed at quickly determining the total content of phenolics in little diluted methanol extracts, we tested an F-C micro-procedure compatible with the final concentration of alcohol equal to $4 \%$. The detail of our procedure is to have used $800 \mu \mathrm{l}$ of a carbonate solution at $5 \%$, carrying out the $\mathrm{F}-\mathrm{C}$ reaction in the presence of $4 \%$ alkali and at $40^{\circ} \mathrm{C}$. In other words, we used an initial carbonate solution with a very low concentration when compared to that used by Singleton, carrying out the assay at a higher final carbonate concentration and a higher temperature than those suggested by Singleton. This procedure allowed us to reduce the reaction time by combining together the effects of alkali and temperature [7].

We hypothesized that the peculiarity of the procedure used in our F-C micro-assay can minimize the interference from alcohol.

The aim of this study is to validate such a hypothesis and to understand why the use of the $5 \%$ carbonate solution is important for the determination of phenolics in the presence of methanol. With this purpose in mind, we have compared the F-C reactions carried out in the presence of different methanol concentrations and derived from two procedures (protocols A and B). In each of these a different carbonate concentration has been used to reach the same alkali amount in the tested reaction mixtures which have the same final volumes.

The F-C reactions were monitored in real-time and the formation of the precipitate was investigated for the first time.

\section{Material and Methods}

\subsection{Reagents}

Experiments were performed using the F-C reagent from Merck (1.09001), sodium carbonate (S6139) and pure caffeic acid (C0626), chosen as a standard phenol, from Sigma. Methanol solutions at the different concentrations each containing $80 \mathrm{mg} / \mathrm{l}$ of caffeic acid, which is the upper limit concentration of the standard that can be used in the assay, were prepared as described in the previous paper [7].

\subsection{Protocols A and B}

The details of protocols A and B are briefly reported here. $100 \mu \mathrm{l}$ of standard solution $(80 \mathrm{mg} / \mathrm{l}$ or 0-blank) at different methanol concentrations were pipetted into separate test tubes and $100 \mu \mathrm{l}$ of F-C reagent were added to each. The mixtures were mixed well and allowed to equilibrate.

For protocol A, after exactly $2 \mathrm{~min}, 800 \mu \mathrm{l}$ of the $5 \%$ $(\mathrm{w} / \mathrm{v})$ sodium carbonate solution were added.

For protocol B, within the $2 \mathrm{~min}, 600 \mu \mathrm{l}$ of distilled water were added and the mixtures were mixed again, then $200 \mu \mathrm{l}$ of the $20 \%(\mathrm{w} / \mathrm{v})$ sodium carbonate solution were added.

The absorbance time-behaviour of investigated mixtures, previously swirled and poured into $1-\mathrm{cm}$ cuvettes, was monitored over a period of $2 \mathrm{~h}$ at $40^{\circ} \mathrm{C}$ recording the absorbance data every two minutes.

\subsection{Apparatus}

The absorbance variations of investigated mixtures were monitored by the spectrophotometer UV/visible ultrospec 4000 (Pharmacia) equipped with a 6 position water heated cell changer.

The morphological observation of some precipitates from both procedures was made by Scanning Electron Microscopy (SEM Zeiss Supra 40).

\section{Results and Discussion}

\subsection{Plan Working}

In order to verify the previously mentioned hypothesis, the following steps were taken to obtain the data used in this study. 
1) The investigated reactions were carried out at $40^{\circ} \mathrm{C}$ and at $4 \%$ carbonate concentration in the final mixture, which corresponds to that of our previous paper [7]. The final carbonate concentration was reached using two different carbonate solutions. The former at 5\%, already employed in our previous paper [7], was the lowest concentration reported in literature, the latter at $20 \%$ was the highest concentration reported in literature $[3,14]$.

2) Only the $20 \%-40 \%-60 \%-80 \%-100 \%$ methanol solutions were investigated to obtain a comprehensive view. Moreover, 3 replicates were carried out on each solution for 4 times to get a fair amount of data.

3) The absorbance time-behaviour of the investigated mixtures was monitored over a period equal to $2 \mathrm{~h}$, which we considered long enough, recording the absorbance data every two minutes, which we considered short enough.

4) To describe the influence of the initial carbonate concentration used (i.e. 5\% carbonate of our procedure) on the F-C micro-method carried out in the presence of methanol, a comparative study of the mean kinetics was made. For reasons of simplicity, the incremental ratios of the first and second order were calculated between adjacent mean absorbance values. The incremental ratio analysis allowed us to determine the beginning and the end of the phenomenon of each mean kinetic with a good degree of approximation. In fact, even though the constant time increments were not infinitely small, they were small enough (two minutes) with respect to the two hours of kinetics so that possible errors in the time determinations are at the most two minutes.

\subsection{Comparison between the Mean Kinetics within Each Procedure}

The overlays of mean kinetics at different methanol concentrations from protocols A and B are showed in Figures 1 and 2, respectively. Mean kinetics at the single methanol concentrations are also showed in these figures to ensure clarity of details.

As expected, the interfering effect of methanol caused the formation of an atypical peak on some reaction curves (Figures 1(a)-(c), Figures 2(a)-(d)), producing an unusual kinetic behaviour. In fact, when the $\mathrm{F}-\mathrm{C}$ reaction was carried out in the presence of methanol, fine solids formed and precipitated in time (clouding and precipitation), outlining the left and the right sides of the peak, respectively. The whole phenomenon appeared spectrophotometrically unhomogeneous, showing high standard deviations (Figures 1(a)-(c), Figures 2(a)-(d)). Moreover, it was rather general with the exception of the kinetics carried out in the presence of $2 \%$ and $4 \%$ methanol from procedure A, where the phenomenon never appeared (Figures 1(d)-(e)), and in the presence of $2 \%$ methanol from pro- cedure B, where only the beginning of the clouding was possible to observe (Figure 2(e)).

On the basis of a comparative analysis among the mean kinetics within each procedure we can claim that both the appearance and the duration of peaks are correlated with methanol concentration. In particular, the peaks of mean kinetics within each procedure shift and the phenomenon duration increases with decreasing methanol concentration (Figures 1 and 2). The behaviours observed within each procedure can be ascribed to the fact that, as the methanol concentration rises, solute (i.e. inorganic salts or ionic species) solubility in reaction mixtures decreases. Theoretically, alcohol affects the dielectric constant of the medium, the inter-ionic attraction, and the solute-solvent interaction [15]. Thus, alcohol can reduce the solubility of inorganic salts decreasing on the one hand the solvation of the ions and on the other the dissociation of salts. In any case, when an alcohol solvent is mixed with a salt water solution, the dielectric property of the newly-formed mixed solvent decreases and the solubility of the solute in the reaction mixture correspondingly decreases [16] bringing about the precipitation phenomenon observed. Furthermore, it was also reported that the presence of alcohol accelerated the particle growth rate [17].

It is important to mention briefly that the precipitate formation ensues from a two-step process. In the first step, called nucleation, some tiny particles known as nuclei are generated. In the second step, called growth, the nuclei grow and the visible solid-phase particles are formed. The rates of these two consecutive steps determine the particle size and its distribution [18]. Moreover, it is worth recalling that, the supersaturation of reaction mixture, the ability to dissolve more solids than that of its equilibrium state, is the primary driving force for the precipitate formation. Generally, a higher degree of supersaturation can generate a rapid nucleation and a slow growth. On the contrary, a lower degree of supersaturation promotes a slow nucleation and a fast growth [19]. It is known that the control of supersaturation requires the knowledge of all the variables affecting the formation of fine solids, such as impurities, temperature, $\mathrm{pH}$ ect. The effects of these parameters on F-C method in the presence of methanol are very interesting and still need to be completely understood.

In this section and in following one, we discussed the results about the effect from the initial carbonate concentration on F-C method in the presence of an increasing methanol concentration in the final reaction mixture.

In the context of this section, the difference observed can be attributed to the fact that, as the methanol concentration rises, the degree of supersaturation of solute in reaction mixtures decreases due to the resulting reduction in 


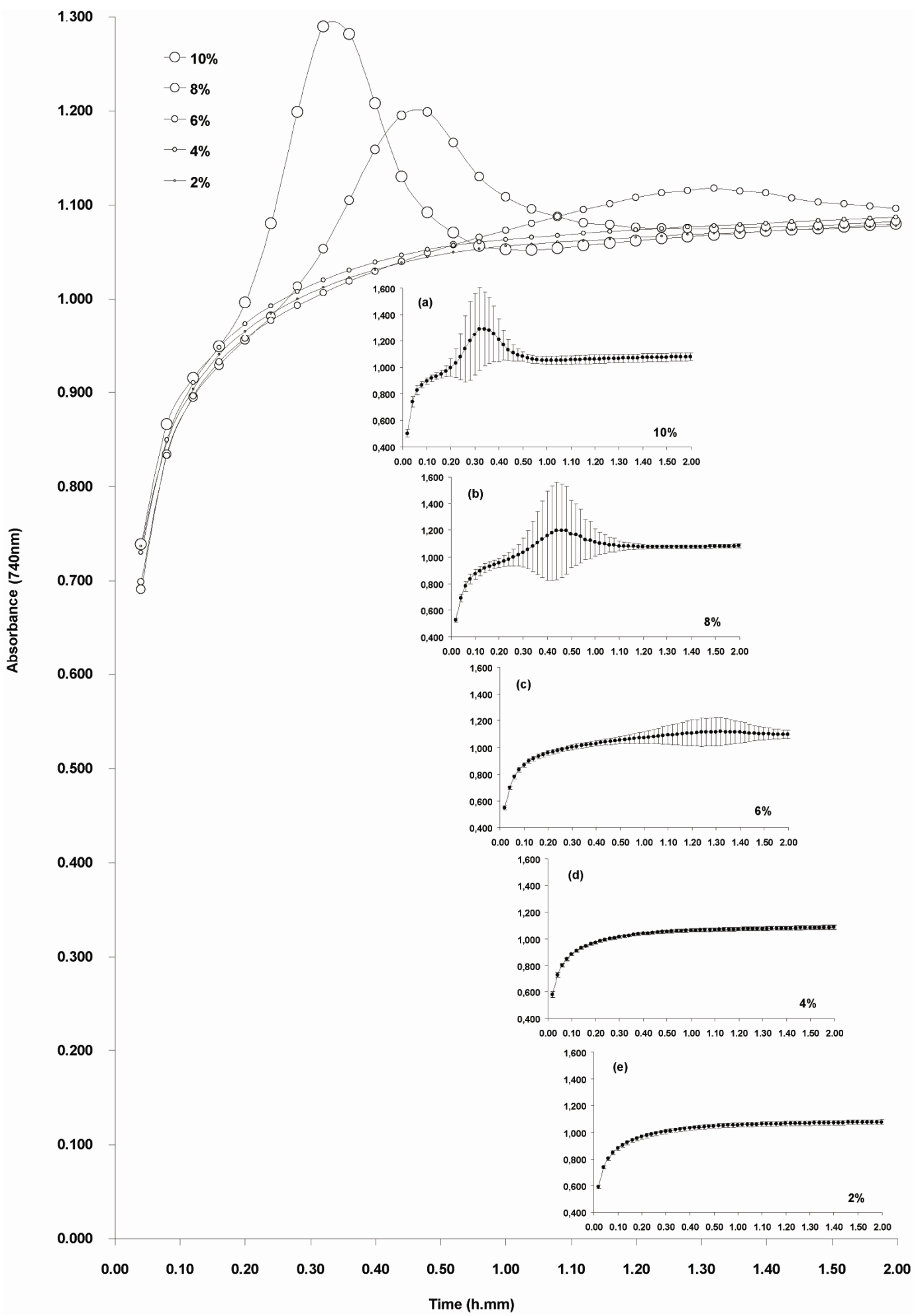

Figure 1. Comparisons between mean kinetics at the different methanol concentrations within protocol A where a $5 \%$ carbonate solution was used to reach the carbonate concentration equal to $4 \%$ in final mixture. Endnotes: the investigated reaction mixtures, each containing $8 \mathrm{mg} / \mathrm{l}$ caffeic acid, were monitored at $40^{\circ} \mathrm{C}$ over a period of $2 \mathrm{~h}$ recording the absorbance data every two minutes. The mean absorbance values and standard deviations of the kinetics carried out in the presence of $10 \%-8 \%-6 \%-4 \%-2 \%$ methanol are shown in the graph inserts a-b-c-d-e, respectively. Instead, only the mean absorbance values recorded every 4 minutes are shown to ensure clarity of details. 


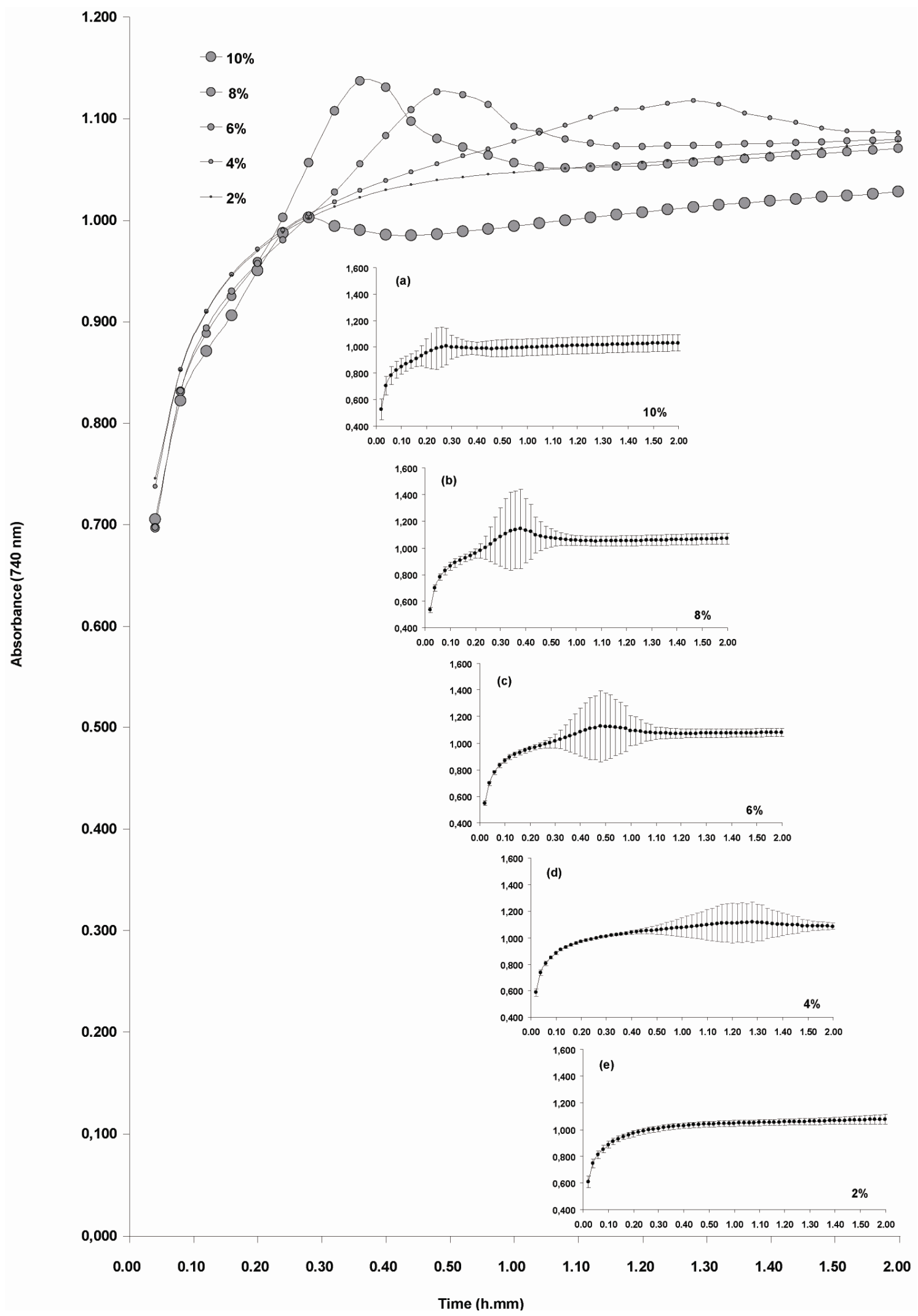

Figure 2. Comparisons between mean kinetics at the different methanol concentrations within protocol B where a $20 \%$ carbonate solution was used to reach the carbonate concentration equal to $4 \%$ in final mixture. Endnotes listed as in Figure 1. 
medium dielectric property. All this makes the phenomenon more and more advanced. This opinion is corroborated by the fact that a lower dielectric constant of mixed solvent corresponds to a lower solubility of inorganic solute and, thus, a shorter induction period for nucleation as well as higher solid particle growth kinetics [16].

Figures 1 and $\mathbf{2}$ show that the maximum absorbance of mean kinetics increases as the methanol concentration increases with the exception of the $10 \%$ methanol concentration from protocol B (Figure 2). In this case, the precipitate has already formed during the reaction exponential phase making the peak very small; furthermore, during the $2 \mathrm{~h}$ period, the colour development is lower and the mean absorbance values show high standard deviations (Figure 2(a)). Presumably, the precipitate formation is so fast that reduces the availability of the ionic species for the development of blue colour.

\subsection{Comparison between the Mean Kinetics from the Two Procedures}

Mean kinetics obtained at the same methanol concentration from the two procedures are showed in Figure 3. Moreover, the data obtained by analyzing the incremental ratios from the two procedures are showed in summary Table 1. In particular, the beginning and the end of the phenomenon, as well as the time where the peak maximum absorbance was reached, are reported. For clarity's sake, the duration of clouding, precipitation and the whole phenomenon are also reported. As can be seen in Table 1 , the duration of the clouding is always shorter than the precipitation one, thus producing asymmetrical peaks (Figure 3).

The thorough comparative analysis of the kinetics allowed us to make the following considerations. The peaks of the kinetics in the presence of $10 \%-8 \%-6 \%$ methanol from protocol A shift with respect to those from protocol B (Figures 3(a)-(c) and Table 1). Moreover, in the whole experiment period, the mean kinetic obtained in the presence of $10 \%$ methanol from protocol $\mathrm{B}$ shows lower absorbance values than those from protocol A (Figure 3(a)), similarly to what was observed among the methanol concentrations within procedure B. Furthermore, the mean kinetics obtained both in the presence of $10 \%$ and $8 \%$ methanol from protocol A show a higher maximum absorbance value than that of the respective mean kinetic from protocol B (Figures 3(a)-(b)), while an opposite behaviour can be observed in the mean kinetic obtained in the presence of $6 \%$ methanol (Figure 3(c)).

Finally, as mentioned in section 3.2, the kinetics obtained in the presence of $4 \%$ and $2 \%$ methanol show no peak when using protocol A (Figures 3(d)-(e) and Table 1).
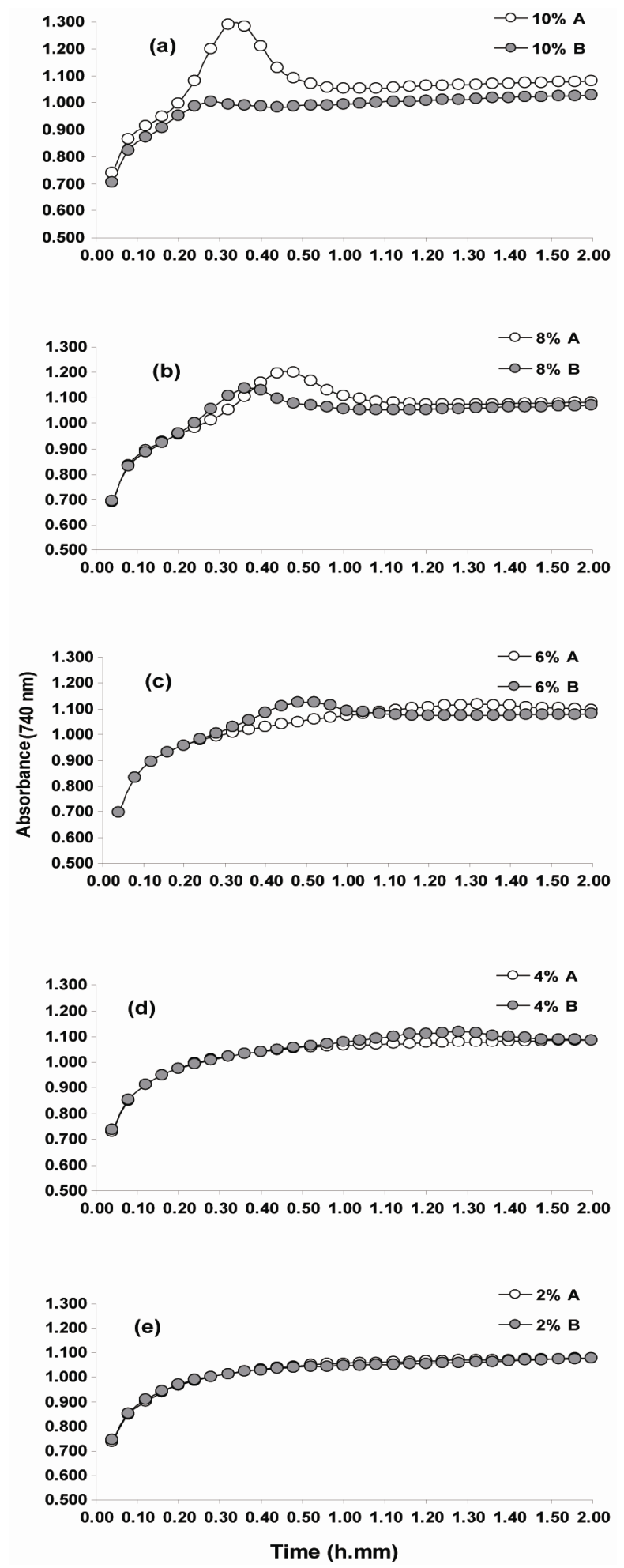

Figure 3. Comparisons between the mean kinetics from the two procedures A (white circles) and B (grey circles). The mean kinetics obtained at the final methanol concentrations equal to $2 \%, 4 \%, 6 \%, 8 \%$ and $10 \%$ were reported in plots (a), (b), (c), (d), (e), respectively. The mean absorbance values are shown over a $2 \mathrm{~h}$ period every $4 \mathrm{~min}$. 
Table 1. Temporal features of the phenomenon due to the methanol effect on the F-C micro-method from procedures A and $\mathrm{B}$, where $5 \%$ and $20 \%$ carbonate as initial concentrations were used respectively.

\begin{tabular}{|c|c|c|c|c|c|c|c|c|c|c|c|}
\hline $\begin{array}{l}\text { Initial carbonate } \\
\text { concentration }(\%)\end{array}$ & & & & 5 & & & & & 20 & & \\
\hline \multirow[t]{3}{*}{$\begin{array}{l}\text { Final methanol } \\
\text { concentration (\%) }\end{array}$} & & 10 & 8 & 6 & 4 & 2 & 10 & 8 & 6 & 4 & 2 \\
\hline & Beginning & $12 \pm 2$ & $18 \pm 2$ & $28 \pm 2$ & - & - & $12 \pm 2$ & $16 \pm 2$ & $20 \pm 2$ & $40 \pm$ & $58 \pm 2$ \\
\hline & Peak maximum absorbance & 34 & 48 & 92 & - & - & 28 & 38 & 48 & 88 & n.d. \\
\hline \multirow[t]{4}{*}{ Time (min) } & End & $60 \pm 2$ & $86 \pm 2$ & n.d. & - & - & $42 \pm 2$ & $68 \pm 2$ & $82 \pm 2$ & n.d. & n.d. \\
\hline & Clouding duration & $22 \pm 2$ & $30 \pm 2$ & $64 \pm 2$ & - & - & $16 \pm 2$ & $22 \pm 2$ & $28 \pm 2$ & $48 \pm 2$ & n.d. \\
\hline & Precipitation duration & $26 \pm 2$ & $38 \pm 2$ & n.d. & - & - & $14 \pm 2$ & $30 \pm 2$ & $34 \pm 2$ & n.d. & n.d. \\
\hline & Phenomenon duration & $48 \pm 4$ & $68 \pm 4$ & n.d. & - & - & $30 \pm 4$ & $52 \pm 4$ & $62 \pm 4$ & n.d. & n.d. \\
\hline
\end{tabular}

Instead, the phenomenon always appears when using protocol $\mathrm{B}$ even if in the presence of $4 \%$ methanol the end of the phenomenon cannot be determined (Table 1), and in the presence of $2 \%$ methanol only the beginning of the clouding can be observed in the $2 \mathrm{~h}$ period (Figure 3(e) and Table 1). On the basis of the results shown in Figure 3 and Table 1 we can assert that the formation of precipitating fine solids is delayed, slowed down and even prevented when using procedure A instead of procedure B.

In our opinion, the differences observed could depend on a different local supersaturation of the ionized species in reaction mixtures probably due to two factors acting simultaneously. The first is the different solvation of carbonate ions in the solutions at $5 \%$ and $20 \%$, used in procedures $\mathrm{A}$ and $\mathrm{B}$, respectively. The second is the different methanol concentration that the compared reaction mixtures have at the moment of carbonate addition. In fact, at that moment, methanol concentration, as well as the $\mathrm{F}-\mathrm{C}$ reagent concentration, in reaction mixtures from procedure $\mathrm{A}$ is higher than those from procedure $\mathrm{B}$.

The results support the idea that, before the reaction equilibrium is reached, the different concentrations of the species involved can give a different diffusion which, in its turn, can cause a different local supersaturation in the compared mixtures from the two procedures. Evidently the local supersaturation is higher in procedure A than procedure B triggering off the formation of many nuclei growing more slowly. Consequently, the phenomenon observed during procedure $\mathrm{A}$ is delayed and slowed down in comparison with that observed during procedure B because smaller particles have formed more slowly.

Our opinion is proved to be right by preliminary SEM analysis which highlights the different precipitate size (Figure 4). Moreover, when using procedure A (5\% car bonate) the phenomenon does not appear at the $2 \%-4 \%$ methanol concentration because the reaction mixture does not become supersaturated at these conditions thanks to the shielding effect of a high solvation degree of the carbonate ions which predominates on the weak effect of methanol due to its low concentration at these conditions. On the contrary, and on the basis of what said above, the evident difference observed between the mean kinetics from both procedures in the presence of $10 \%$ methanol can be due to the fact that the experiments conducted using procedure B led to an early and fast formation of bigger fine solids which precipitate more quickly than those obtained when using procedure A.

Therefore, we can conclude that, although the same carbonate amount is reached in final mixtures, the initial carbonate concentration plays an important role in the $\mathrm{F}-\mathrm{C}$ reaction when this is carried out in the presence of methanol since the generation of nuclei and their growth is based on the local properties of mixture, rather than the average properties.

\subsection{Observation of Precipitate}

After the $2 \mathrm{~h}$ period, by carefully observing the tested mixtures in each cuvette, we remarked that the precipitates were quite evidently visible to the naked eye when carrying out the $\mathrm{F}-\mathrm{C}$ reaction in the presence of $10 \%$ and $8 \%$ methanol in both procedures, even if the precipitate from procedure $\mathrm{B}$ was slightly higher than that from procedure A. Instead, when the $\mathrm{F}-\mathrm{C}$ reaction was conducted in the presence of $6 \%$ methanol using protocol $\mathrm{B}$, the precipitates were less visible and not always visible using protocol A. In this latter case the peaks did not appear, either. In accordance with this behaviour, the precipitates were not always observed in the presence of $4 \%$ and $2 \%$ methanol when using protocol $\mathrm{B}$ and never observed when using protocol A. 

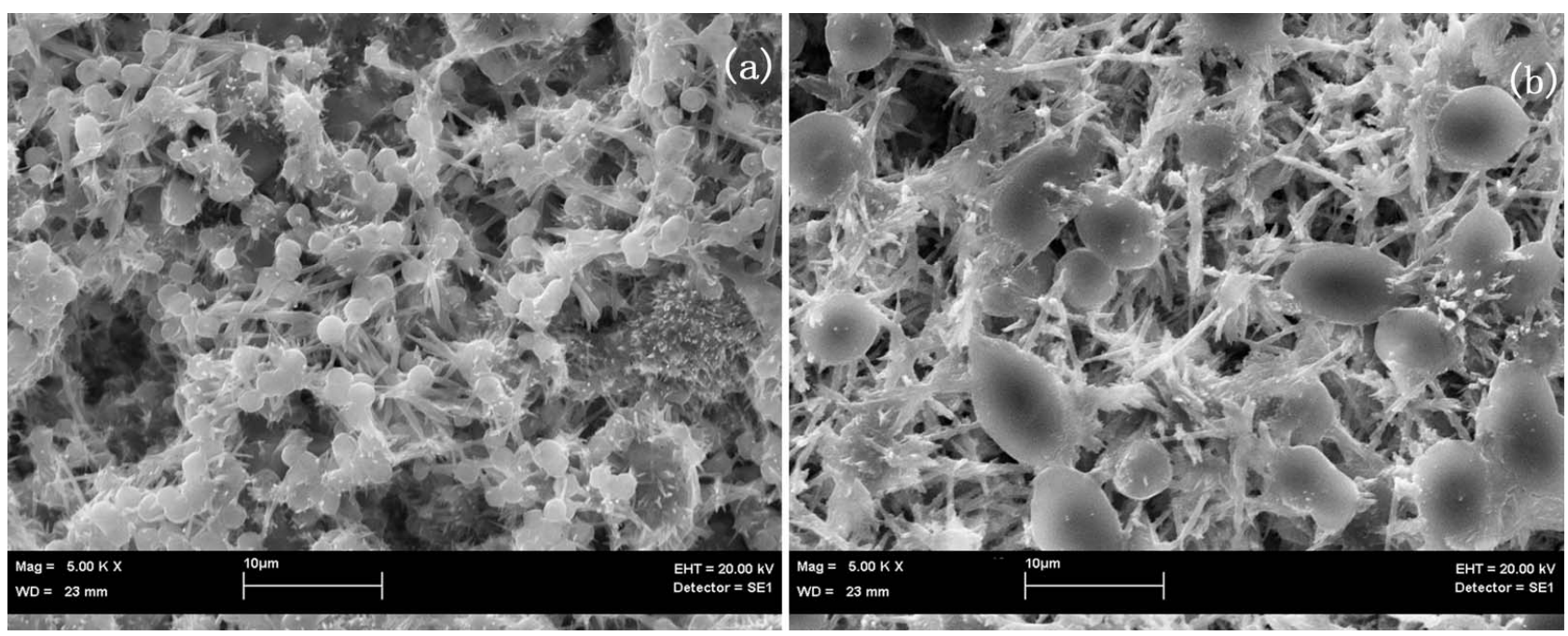

Figure 4. SEM images of precipitates obtained during F-C reaction carried out in the presence of $6 \%$ methanol from the procedures $A(a)$ and $B(b)$.

On the basis of these observations, we can assert that the yield of precipitation is strictly related to the stage of precipitation which, in its turn, depends on supersaturation degree. Nonetheless, the data of this study did not allow us to clarify why the phenomenon is random in the presence of $6 \%$ methanol when using protocol $\mathrm{A}$ as well as $4 \%$ and $2 \%$ methanol when using protocol $\mathrm{B}$.

The precipitates obtained in the presence of $6 \%$ methanol from both procedures were also observed by scanning electron microscope (SEM). SEM images, reported in Figure 4, show that presumably the precipitate consists both of crystalline and amorphous material. The former is mostly represented by spindle-shaped crystals which were bigger (about equal to and smaller than 10 $\mu \mathrm{m})$ in precipitate from procedure B than those from procedure A (smaller than $5 \mu \mathrm{m}$ ). The latter is mostly represented by spherical particles with a mixture of some ellipse-like particles. In any case, both the spherical particles and ellipse-like particles showed a mean bigger diameter (about equal to and smaller than $10 \mu \mathrm{m}$ ) in the precipitate from procedure $B$ than that from procedure $A$ (about equal to and smaller than $3 \mu \mathrm{m}$ ). Moreover, some of the observed particles were attached to each other forming agglomerates.

\subsection{Important Detail from Real Time Reaction Monitoring}

In accordance with the previous paper, our experiments indicated that procedure A allows us to carry out without any problem the $\mathrm{F}-\mathrm{C}$ micro-method in the presence of methanol up to the $4 \%$ concentration and, contrary to what we expected, the precipitate never appeared at this methanol concentration in the $2 \mathrm{~h}$ period. Surprisingly, the results from procedure A also showed that a methanol concentration equal to $6 \%$ can be reached in the final mixture, as a new extreme condition, by making the real time reading at $24 \mathrm{~min}$. In fact, the mean absorbance value at these conditions $(\mathrm{Abs}=0.977 \pm 0.019)$ was similar to that found in the presence of $4 \%$ methanol at $20 \min (\mathrm{Abs}=0.973 \pm 0.008)$ corresponding to about $96 \%$ of blue colour development, taken as reference absorbance [7]. Moreover, by real time reading at $24 \mathrm{~min}$ the F-C assay can still be carried out quickly, given that neither filtration nor centrifugation techniques are necessary because precipitates will appear later (Table 1).

\subsection{Conclusions}

The carbonate concentration used in the F-C micromethod, carried out in the presence of methanol, is an important parameter which should not be ignored since it can modify the formation of precipitating fine solids due to the interfering effect of methanol. The data presented in this manuscript not only show in depth the methanol effect on the F-C reactions but also highlight why the initial carbonate concentration influences it. In particular, the formation of particles is delayed and slowed down with decreasing methanol concentration and can be further delayed, slowed down and even prevented when a carbonate solution at $5 \%$ instead of $20 \%$ is used. The current investigation, validating our hypothesis, allowed us to conclude that the change of ionic species (reagent and/or product) solubility in the F-C reaction is correlated to concentrations both of methanol (concentration in final mixture) and carbonate (initial concentration of stock solution). Moreover, a local high supersaturation can be formed when using procedure A instead of pro- 
cedure $\mathrm{B}$ resulting in a higher formation of nuclei growing more slowly.

Furthermore, we can assert that F-C assay can be carried out in the presence of $6 \%$ methanol, as a new extreme concentration, by real time reading the absorbance at $24 \mathrm{~min}$.

In our opinion, the real time monitoring of absorbance can be a useful means to explore the effect of other parameters on precipitate formation caused by interference from methanol in the $\mathrm{F}-\mathrm{C}$ reaction.

The results of this study provide important information for further investigations aimed at increasing the alcohol concentration in the final F-C reaction mixture.

\section{Acknowledgements}

The authors wish to acknowledge Dr. Luca Medici and Dr. Antonio Lettino for their scientific and technical assistance in SEM analysis.

\section{References}

[1] N. C. Cook and S. Samman, "Flavonoids-Chemistry, Metabolism, Cardioprotective Effects and Dietary sources," The Journal of Nutritional Biochemistry, Vol. 7, 1996, pp. 66-76. doi:10.1016/0955-2863(95)00168-9

[2] C. A. Rice-Evans, R. N. J. Mille and G. Paganga, “AntiOxidant Properties of Phenolic Compounds," Trends in Plant Science, Vol. 2, 1997, pp. 152-159. doi:10.1016/S1360-1385(97)01018-2

[3] V. L. Singleton and J. A. Rossi, "Colorimetry of Total Phenolics with Phosphomolybdic-Phosphotungstic Acid Reagents," American Journal of Enology and Viticulture, Vol. 16, 1965, pp. 144-158.

[4] J. Blouin, L. LLorca, F. R. Montreau and J. H. Dufour, "Etude des Conditions Optimales Pour la Determination des Composes Phenoliques Totaux par le Reactif de Folin Ciocalteu," Connaissance de la Vigne et du Vin, Vol. 6, 1972, pp. 405-413.

[5] F. R. Montreau, "Sur le Dosage des Composes Phenoliques Totaux dans les vins par la Methode F-C," Connaissance de la Vigne et du Vin, Vol. 6, 1972, pp. 397-404.

[6] K. Slinkard and V. L. Singleton, "Total Phenol Analysis: Automation and Comparison with Manual Methods," American Journal of Enology and Viticulture, Vol. 28, 1977, pp. 49-55.

[7] N. Cicco, M. T. Lanorte, M. Paraggio, M. Viaggiano and V. Lattanzio, "A Reproducible Rapid and Inexpensive Folin-Ciocalteu Micro-Method in Determining Phenolics of Plant Methanol Extracts," Microchemical Journal, Vol. 91, No. 1, 2009, pp. 107-110. doi:10.1016/j.microc.2008.08.011
[8] O. S. Balogun and T. Teraoka, "Time-Course Analysis of the Accumulation of Phenols in Tomato Seedlings Infected with Potato Virus $X$ and Tobacco mosaic virus," Biokem, Vol. 16, No. 2, 2004, pp. 112-120.

[9] M. Monagas, V. Nunez, B. Bartolomé and C. Gomez-Cordoves, "Phenolic Content of Blends of Tempranillo with Graciano or Cabernet Sauvignon Wines Produced in Spain," Food Technology and Biotechnology, Vol. 44, No. 4, 2006, pp. 507-513.

[10] V. Papadimitriou, T. G. Sotiroudis, A. Xenakis, N. Sofikiti, V. Stavyiannoudaki and N. A. Chaniotakis, "Oxidative Stability and Radical Scavenging Activity of Extra Virgin Olive Oils: An Electron Paramagnetic Resonance Spectroscopy Study," Analytica Chimica Acta, Vol. 573574, 2006, pp. 453-458. doi:10.1016/j.aca.2006.02.007

[11] G. Marigo, "Sur une Methode de Fractionnement et d'Estimation des Composes Phenoliques Chez lez Vegetaux," Analusis, Vol. 2, 1973, pp. 106-110.

[12] G. A. Spanos and R. E. Wrolstad, "Influence of Processing and Storage on the Phenolic Composition of Thompson Seedless Grape Juice," Journal of Agricultural and Food Chemistry, Vol. 38, No. 7, 1990, pp. 1565-1571. doi:10.1021/jf00097a030

[13] N. Sadler and H. Jacobs, "Application of the Folin-Ciocalteu Reagent to the Determination of Salbutamol in Pharmaceutical Preparations," Talanta, Vol. 42, No. 10, 1995 , pp. 1385-1388. doi:10.1016/0039-9140(95)01534-I

[14] V. L. Singleton, R. O. Orthofer and R. M. Lamuela-Raventos, "Analysis of Total Phenols and Other Oxidation Substrates and Antioxidants by Means of Folin-Ciocalteu Reagent," Methods in Enzymology, Vol. 299, 1999, pp. 152-178. doi:10.1016/S0076-6879(99)99017-1

[15] F. Manoli and E. Dalas, "Spontaneous Precipitation of Calcium Carbonate in the Presence of Ethanol, Isopropanol and Diethylene Glycol," Journal of Crystal Growth, Vol. 218, No. 2-4, 2000, pp. 359-364. doi:10.1016/S0022-0248(00)00560-1

[16] M. Z. C. Hu, E. A. Payzant and C. H. Byers, "Sol-Gel and Ultrafine Particle Formation via Dielectric Tuning of Inorganic Salt-Alcohol-Water Solutions," Journal of Colloid and Interface Science, Vol. 222, 2000, pp. 20-36.

[17] G. Guo, G. Yan, L. Wang and J. Huang, "Crystallization of Strontium Carbonate in Alcohol or Water Solution Containing Mixed Nonionic/Anionic Surfactants," Materials Letters, Vol. 62, No. 24, 2008, pp. 4018-4021. doi:10.1016/j.matlet.2008.05.052

[18] A. S. Myerson, "Crystal, Crystal Growth and Nucleation," Handbook of Industrial Crystallization, Butterworth-Heinmann Publications, London, 1993, pp. 33-63.

[19] V. Liotta and V. Sabesan, "Monitoring and Feedback Control of Supersaturation Using ATR-FTIR to Produce an Active Pharmaceutical Ingredient of a Desired Crystal Size," Organic Process Research \& Development, Vol. 8, No. 3, 2004, pp. 488-494. doi:10.1021/op049959n 